\title{
Evaluación de una propuesta para la enseñanza y el aprendizaje de la astronomía en secundaria
}

\section{Evaluation of an instruction for teaching and learning of astronomy in secondary school}

\author{
Rafael Palomar \\ Colegio El Prat. LLíria. España. \\ rafa.palomar@gmail.com \\ Jordi Solbes \\ Departamento de didáctica de las ciencias. Universitat de València. España \\ Jordi.solbes@uv.es
}

RESUMEN • Este trabajo de investigación presenta una propuesta para la enseñanza de la astronomía enmarcada en el socioconstructivismo que se ha implementado en alumnos de secundaria de 17 años, realizando un diseño de tipo pre-test y post-test. El análisis de los datos ha revelado la propuesta como muy eficaz, mostrando una mejora significativa en prácticamente todos los ítems analizados. También se ha mostrado que, a pesar de partir con conocimientos astronómicos similares, los alumnos de la modalidad científico-tecnológica han obtenido una mejora significativamente mayor que los de la opción de humanidades-ciencias sociales.

PALABRAS CLAVE: astronomía; astrodidáctica; ciencias para el mundo contemporáneo; evaluación; investigación en el aula.

ABSTRACT - This research shows a proposal to teach astronomy -framed in the socio-constructivism which has been implemented in 17-year-old students in secondary, carrying out a design of pre-test and post-test type. The data analysis has revealed it as a very effective treatment, showing a significant improvement in almost all items tested. It has also been shown that despite similar astronomical knowledge from, the students of the scientific-technological option have obtained a significantly greater improvement than those of the humanities and social-sciences option.

KEYWORDS: astronomy; astrodidactic; science for the contemporary world; evaluation; classroom research. 


\section{INTRODUCCIÓN}

Como se ha mostrado en otras investigaciones (Comins, 1993; Hansson, 2006; Trumper, 2001; Solbes y Palomar, 2013) los y las estudiantes no comprenden y/o desconocen aspectos básicos de la astronomía, pese a la reiterada enseñanza de esta. La mayoría de las investigaciones de astrodidáctica se centran en el sistema Tierra-Sol-Luna (Dove, 2002; Trundle, Atwood y Chistopher, 2007) y mucho menos en aportaciones astronómicas más recientes (Pasachoff, 2001). Otras investigaciones se centran en la formación del profesorado de todos los niveles, desvelando que tampoco es adecuada en estos temas (Camino, 1995; Atwood y Atwood, 1997; Parker y Heywood, 1998; Vega, 2001; Ogan-Bekiroglu, 2007; Mulholland y Ginns, 2008).

Este trabajo es la continuación de una investigación (Solbes y Palomar, 2013) en la que se evaluaron los conocimientos astronómicos de un grupo de estudiantes de $1 .^{\circ}$ de bachillerato (17 años) que han cursado Ciencias para el Mundo Contemporáneo (CMC). Esta materia permite realizar una enseñanza divulgativa y contextualizada de la ciencia a todos los estudiantes de bachillerato, sea cual sea su especialidad, e introducir contenidos de astronomía (el origen del universo, la génesis de los elementos, la exploración del sistema solar). Los resultados obtenidos fueron muy bajos y el análisis detallado de estos nos permite detectar una serie de dificultades de aprendizaje (Solbes, 2009) que se pueden ver en la tabla 1 vinculados a los objetivos de aprendizaje que pretende la propuesta que presentaremos a continuación. Además, en el análisis realizado en dicho trabajo, se constata que la introducción de la astronomía en los textos más utilizados de CMC no tiene en cuenta las dificultades de los estudiantes. En concreto, dicha introducción se realiza de una forma muy teórica y verbalista y sin mostrar las observaciones que han permitido comprobar los enunciados astronómicos, sin tener en cuenta la escasa comprensión que tienen los estudiantes de aspectos astronómicos básicos del sistema Sol-Tierra-Luna insistentemente estudiados en primaria. Tampoco se trabajan suficientemente las dimensiones y tiempos astronómicos, muy dificultosos para el alumnado dado que superan con mucho la escala humana, ni cuestiones que permitan desarrollar competencias críticas. Y aunque sí aparecen las relaciones de la astronomía con la tecnología, no se mencionan apenas las aplicaciones básicas de la astronomía necesarias para la supervivencia de la especie.

Por ello nos planteamos el problema de comprobar si una propuesta de actividades, elaborada teniendo en cuenta el cuerpo de conocimientos de la didáctica de las ciencias, puede mejorar el aprendizaje de la astronomía en el bachillerato superando las dificultades detectadas en los alumnos. También nos preguntamos si influye en los resultados la modalidad de bachillerato de la que proceden los alumnos.

\section{MARCO TEÓRICO Y PROPUESTA DE ENSEŃANZA}

A mediados de los ochenta, se constata un descenso del interés de los alumnos hacia el aprendizaje de las ciencias (en especial, la física y la química) y, en consecuencia, un abandono de su estudio. Por ello, las propuestas de los años noventa proponen conseguir no solo cambios conceptuales y procedimentales, sino también axiológicos y actitudinales. Sin embargo, en trabajos recientes se constata que ese desinterés no ha hecho más que aumentar por causas como la imagen pública de la física, la forma en que se enseña y por problemas de género (Lima, Lang Da Silveira y Ostermann, 2012; Solbes, Montserrat y Furió, 2007; Solbes y Vilches, 1997). Así, se hace necesario combatir ese desinterés, promoviendo una visión socioconstructivista, con enfoques de cambio conceptual, procedimental y actitudinal y axiológico, con la inclusión de contenidos de historia de la ciencia e interacciones CTS (Solbes, Montserrat y Furió, 2007; Solbes y Vilches, 1997), y mediante el uso de programas de actividades. 
Esto nos ha llevado a desarrollar una propuesta para la enseñanza de la astronomía en CMC que puede consultarse en la página web <www.astrodidactica.com> en el apartado "Propuesta CMC» y cuyos objetivos se detallan en la tabla 1, vinculándolos a las dificultades de aprendizaje antes mencionadas. Además, una tercera columna indica los ítems del cuestionario utilizado para valorar la propuesta, cuyos enunciados aparecen explicitados más adelante, en la tabla 2, y descritos con más detalle en Solbes y Palomar (2013).

Tabla 1.

Objetivos y dificultades relacionados con los ítems del cuestionario de alumnos

\begin{tabular}{|c|c|c|}
\hline Objetivos & Dificultades & Ítems \\
\hline $\begin{array}{l}\text { 1. Comprender aplicaciones básicas de la astronomía } \\
\text { y su importancia para la supervivencia de la especie. }\end{array}$ & $\begin{array}{l}\text { Como las sociedades avanzadas viven al margen de la } \\
\text { naturaleza, no tienen claro el papel de la astronomía en } \\
\text { la orientación, agricultura, etc. }\end{array}$ & $\begin{array}{l}1 \\
3\end{array}$ \\
\hline $\begin{array}{l}\text { 2. Familiarizarse con los métodos de trabajo de la as- } \\
\text { tronomía de observación visual (observaciones as- } \\
\text { tronómicas diurnas y nocturnas, gnomon, etc.). }\end{array}$ & $\begin{array}{l}\text { Dado que la mayoría de la población vive en ciudades } \\
\text { no puede disfrutar el cielo nocturno, y así desconocen } \\
\text { los procedimientos implicados en la observación. }\end{array}$ & $\begin{array}{l}1 \\
3\end{array}$ \\
\hline $\begin{array}{l}\text { 3. Explicar observaciones del sistema Tierra-Sol-Luna } \\
\text { (las estaciones, las fases de la Luna y las horas en las } \\
\text { que se observa, etc.). }\end{array}$ & $\begin{array}{l}\text { El pensamiento basado en la percepción y la dificultad } \\
\text { ligada al cambio de sistema de referencia (SR) en que se } \\
\text { representan las posiciones de la Luna (sobre la eclíptica) } \\
\text { al SR en que se realizan las observaciones (la superficie } \\
\text { terrestre). }\end{array}$ & $\begin{array}{l}4 \\
5\end{array}$ \\
\hline $\begin{array}{l}\text { 4. Familiarizar a los alumnos con la naturaleza de la } \\
\text { ciencia, que elabora modelos para explicar los pro- } \\
\text { blemas hasta que surgen dificultades que obligan a } \\
\text { cambiarlos, en este caso, el geocéntrico, heliocéntri- } \\
\text { co, newtoniano, etc. }\end{array}$ & $\begin{array}{l}\text { No comprenden que algunos enunciados solo cobran } \\
\text { sentido en un determinado modelo ni los grandes } \\
\text { avances que suponen (la gravitación universal rompe } \\
\text { la barrera cielo-Tierra, el heliocentrismo nuestra que la } \\
\text { Tierra no es un SR privilegiado, etc.). }\end{array}$ & $\begin{array}{l}2 \\
6\end{array}$ \\
\hline 5. Modelizar el sistema solar a escala. & $\begin{array}{l}\text { Supera con mucho la escala humana y en prácticamente } \\
\text { ningún sitio existen representaciones adecuadas. }\end{array}$ & 7 \\
\hline $\begin{array}{l}\text { 6. Comprender, a partir de observaciones astronómi- } \\
\text { cas, que vivimos en una galaxia y su forma aproxi- } \\
\text { mada. }\end{array}$ & $\begin{array}{l}\text { De nuevo la dificultad que entraña el cambio de SR } \\
\text { para dar sentido a una observación puede hacer que no } \\
\text { tengan claro que la Vía Láctea es el plano de la galaxia. }\end{array}$ & 8 \\
\hline $\begin{array}{l}\text { 7. Valorar la importancia de las relaciones de la astro- } \\
\text { nomía con la tecnología y la sociedad. }\end{array}$ & $\begin{array}{l}\text { No es fácil conectar bidireccionalmente la astronomía } \\
\text { con otras ciencias, con la tecnología y con la sociedad. }\end{array}$ & 9 \\
\hline $\begin{array}{l}\text { 8. Comprender que hay diversos tipos de estrellas que } \\
\text { evolucionan y que juegan un importante papel en } \\
\text { la vida del Universo. }\end{array}$ & $\begin{array}{l}\text { Concepción estática y no evolutiva de las estrellas, debi- } \\
\text { do a las grandes escalas temporales implicadas. }\end{array}$ & - \\
\hline $\begin{array}{l}\text { 9. Comprender la teoría del Big Bang y las pruebas } \\
\text { de esta, así como que requiere refinamientos para } \\
\text { explicar nuevas observaciones. }\end{array}$ & $\begin{array}{l}\text { La abstracción que supone considerar la creación del } \\
\text { espacio y del tiempo junto a la de la materia. }\end{array}$ & $\begin{array}{l}10 \\
11\end{array}$ \\
\hline $\begin{array}{l}\text { 10. Comprender que el Universo está formado por mi- } \\
\text { les de millones de galaxias, ninguna de las cuales } \\
\text { ocupa un lugar central; a gran escala es homogéneo } \\
\text { e isótropo. }\end{array}$ & Superan con mucho la escala humana. & - \\
\hline $\begin{array}{l}\text { 11. Valorar la contribución de la astronomía al pensa- } \\
\text { miento crítico. }\end{array}$ & $\begin{array}{l}\text { No ser conscientes de que las verdades científicas tienen } \\
\text { que luchar contra los poderes y concepciones estableci- } \\
\text { dos y que muchos enunciados supuestamente científi- } \\
\text { cos no se pueden probar (la influencia de los astros en la } \\
\text { vida humana, la existencia de los OVNI, etc.). }\end{array}$ & $\begin{array}{r}12 \\
6\end{array}$ \\
\hline
\end{tabular}


Los contenidos conceptuales, procedimentales y actitudinales de nuestra propuesta se desarrollan atendiendo a los siguientes criterios:

Los conceptos se abordan mostrando una imagen de la ciencia contextualizada, en toda su riqueza y complejidad (Solbes y Palomar, 2011):

- Se aprovechan los acontecimientos históricos para una mayor comprensión de los conocimientos científicos, y se consideran los problemas planteados que llevaron a la construcción de dichos conocimientos. Muy en particular, aproximándonos al surgimiento de un nuevo paradigma, basado en unas mismas leyes para todo el universo y fruto del trabajo de muchas personas (Copérnico, Kepler, Galileo, Newton y un largo etcétera), que unificaba la mecánica terrestre y celeste, poniendo fin a una de las barreras que había impedido el avance científico a lo largo de más de veinte siglos. De esa manera se contribuye a superar las dificultades 4 y 11 , señaladas en la tabla 2.

- Buscando superar las dificultades 8 y 9 se recogen actividades encaminadas a mostrar conceptos de física estelar, para evitar considerar las estrellas como objetos inmutables, así como conceptos de cosmología que hagan comprender el Universo como un todo, su nacimiento y evolución según las pruebas de la teoría del Big Bang.

- Se incluyen actividades de escala, espaciales y temporales, que familiaricen a los estudiantes con números muy alejados de la escala humana, para tratar de superar las dificultades 5 y 10 . Dichas actividades deben facilitar el cambio del universo de sentido común centrado en la Tierra, con un millar de estrellas «fijas», tan pequeño como el actual sistema solar y "creado» hace unos 6.000 años, a un universo poblado por $10^{11}$ galaxias, con un tamaño de $10^{26} \mathrm{~m}$ y una duración de 13.700 millones de ańos aproximadamente (Cardenete, 2009; Causeret, Fouquet y SarrazinVilas, 2008).

En relación con los procedimientos hay que tener en cuenta que la astronomía es una ciencia observacional y olvidarlo puede ofrecer una visión deformada de esta:

- Se plantean actividades de astronomía diurna mediante la utilización del gnomon (Grup Astre, 1998) o del reloj de Sol (Arribas, 2001), con el fin de superar la dificultad número 2.

- También se plantean actividades de astronomía nocturna si la contaminación lumínica lo permite (Zuza y Alduncin, 2009), mediante la observación de las fases de la Luna o el uso del planisferio celeste. Resulta conveniente, además, utilizar alguna aplicación para móviles de localización estelar y/o búsqueda de satélites y estación espacial y, así, contribuir a superar la dificultad 7.

- Las observaciones dan pie a que comprendan los fenómenos del sistema Tierra-Sol-Luna, a pesar de la dificultad en el cambio del sistema de referencia, o también que concluyan que vivimos en una galaxia. De esta forma, las observaciones contribuyen a superar las dificultades 3 y 6 .

- Por último, la historia de las ciencias familiariza con la Naturaleza de la Ciencia (Jiménez Liso, López-Gay y Martínez Chico, 2012), al poner de manifiesto que la ciencia es un conjunto articulado de teorías o modelos que interpretan la realidad; cuando estos modelos no pueden explicar ciertos problemas, son sustituidos por otros nuevos. Así, se contribuye a superar la dificultad número 4.

Por último, con respecto a las actitudes y valores, dado que la astronomía es la rama de la ciencia con más aficionados no profesionales, posiblemente porque trata problemas de interés para los seres humanos, algunas actividades tratan de despertar el interés acerca de los distintos cuerpos del sistema solar y del universo, sus relaciones, cómo ha evolucionado históricamente la concepción del universo, la utilidad y las repercusiones que tiene la astronomía en el ámbito tecnológico, etc. Más en concreto: 
- Se realizan actividades de relaciones CTS que ponen de manifiesto que las ciencias están sometidas a ciertos condicionantes y determinadas por la sociedad en la que se desarrollan, para superar la dificultad 7.

- Parte de las actividades diseñadas van en la línea de superar la dificultad 1, y hacer comprender las aplicaciones básicas de la astronomía, puesto que actualmente se vive de espaldas a la naturaleza y los estudiantes olvidan el papel de la astronomía en la orientación, agricultura, etc.

- Por último, para superar la dificultad 11, nuestra propuesta fomenta el pensamiento crítico, cuestionando las pseudociencias como la astrología o la ufología, mostrando la lucha entre las verdades científicas y el poder establecido y objetando contra el eurocentrismo. Además, destaca el papel de las mujeres en la historia de la astronomía (Pérez Sedeño, 2003) y también muestra la relación de la tecnología y la astronomía (Solbes y Vilches, 1997).

En resumen, la propuesta permite presentar una imagen más contextualizada de la ciencia y que así su enseñanza se transforme en un elemento fundamental para la formación de los ciudadanos, no solo para su capacitación profesional, sino también para que puedan comprender, juzgar y tomar decisiones en los temas científicos y tecnológicos objetivos de las CMC.

\section{Ejemplos de actividades}

El programa de actividades se encuentra desarrollado en Palomar (2013), y además, la propuesta aparece publicada en la sección «Propuesta $\mathrm{CMC}$ » de la página web de uno de los autores <www.astrodidactica.com>, una página que recoge propuestas y materiales de enseñanza de la astronomía. A modo de ejemplo se enuncian y comentan algunas de estas actividades, las dos primeras procedimentales, la tercera CTS y las restantes conceptuales:

Actividad. Realiza una observación nocturna de corta duración. Debe realizarse el dibujo de al menos dos agrupaciones de estrellas para tratar de identificarlas el próximo día en la clase con la ayuda de un planisferio. Es necesario anotar la hora de la observación. En el caso de observar algún objeto llamativo en el cielo también conviene anotarlo.

El primer resultado que cabe esperar de muchos estudiantes en esta actividad es la dificultad de realizar observaciones en la gran mayoría de nuestras poblaciones. Ello puede dar pie al inicio de una discusión acerca de los problemas que plantea la contaminación atmosférica y, muy particularmente, la lumínica, que nos está privando literalmente del paisaje celeste, además de afectar a los ciclos vitales de las plantas y los animales que viven en las ciudades, incluidos los seres humanos. Se trata de un aspecto sobre el que incidiremos en otra actividad.

Actividad. Utilizando la aplicación libre Stellarium sitúate en el tiempo y lugar de los antiguos egipcios (año 2780 a. n. e., antigua ciudad de Menfis: $29^{\circ} 58^{\prime} 33.744^{\prime} \mathrm{N} ; 31^{\circ} 7^{\prime} 49.476^{\prime} \mathrm{E}$ ) y localiza el orto helíaco (momento en el que una estrella comienza a ser visible inmediatamente antes de que salga el Sol) de la estrella Sirio.

Una de las aplicaciones de la astronomía a lo largo de la historia es la creación de calendarios, y conviene poner de manifiesto cómo las regularidades en los fenómenos celestes han contribuido al desarrollo de la civilización. Un caso particular es el de los egipcios, que fijaron el inicio de su calendario (del cual proviene el nuestro) en el orto helíaco de la estrella Sirio. Esta actividad muestra la aplicación de la astronomía en la vida civil y en la agricultura de los pueblos antiguos, a la vez que enseña al estudiante el uso de un programa libre de simulación astronómica, Stellarium, disponible en la página web <http://www.stellarium.org/es/>. 
Actividad. ¿Qué hechos y razones conoces que pongan de manifiesto el carácter acientífico de la astrología?

Es preciso denunciar el carácter anticientífico de estas creencias, que siguen siendo avaladas por algunos medios de comunicación que publican horóscopos cada semana. Puede realizarse alguna actividad dirigida a que el alumno comprenda el carácter acientífico de la astrología. Por ejemplo, días antes, se solicita el nombre de los estudiantes, así como la fecha y hora de nacimiento, con el pretexto de que un astrólogo va a realizarles la carta astral personalizada. Se preparan los sobres personalizados con el nombre del alumno y en el interior se coloca el texto utilizado por el psicólogo B. R. Forer (1949) para mostrar que una descripción suficientemente ambigua encaja con cualquier persona. Al entregar a los alumnos, y una vez estos han leído para sí mismos el texto, se les pide que puntúen el grado de acierto en una escala de 1 a 5 (la puntuación en el estudio original fue de 4,26). Ahora ya pueden leer el texto en voz alta y darse cuenta de que todos tienen el mismo texto, que, con seguridad, tan acertado había estado.

Actividad. Los antiguos griegos pensaban que la Tierra era el centro del universo, estaba inmóvil, y que el Sol y el resto de los astros se movían a su alrededor. ¿En qué se basaban para pensar así, además de en sus observaciones astronómicas?

Algunos estudiantes se refieren a la salida y puesta de las estrellas, o a otras experiencias cotidianas como, muy particularmente, el hecho de que no notemos el movimiento de la Tierra. El reposo de la Tierra parecía, efectivamente, como algo «evidente» e incuestionable y estaba relacionado con otras evidencias "de sentido común», como la tendencia al reposo de los objetos terrestres, mientras los astros giraban indefinidamente (respecto a si la Tierra permanece inmóvil o no, el visionado de un fragmento de la película Ágora, disponible en línea en <http://youtu.be/gtSD8ShDxEc>, en la que Hypatia se realiza esta misma pregunta, puede arrojar algo de luz a la cuestión). La aceptación general de este sistema geocéntrico se mostraba muy claramente en el mismo lenguaje ordinario, lleno de expresiones como, por ejemplo, «el Sol sale por...». Los estudiantes comprenden así que estas ideas no eran descabelladas, sino que se apoyaban, como hemos visto, en experiencias de la vida cotidiana.

Actividad. En 1929 Hubble descubrió que la luz procedente de las galaxias lejanas tiene frecuencias inferiores a las correspondientes al espectro del Sol. Es decir, hay un desplazamiento hacia el rojo tanto mayor cuanto más lejos están las galaxias. ¿¿ué explicación puede darse de este hecho teniendo en cuenta el diferente tono en la sirena de una ambulancia o en el silbido de un tren cuando se acerca o cuando se aleja?

Muchos estudiantes han observado el efecto Doppler con ondas sonoras. Conviene que comprendan que cuando se acercan el tono es más agudo (lo que corresponde a una frecuencia mayor) y cuando se alejan es más grave (frecuencia menor). Como las líneas espectrales de dichas galaxias estaban desplazadas hacia el rojo, es decir, hacia frecuencias menores (o longitudes de onda mayores), esto demostró que las galaxias se alejaban de la Tierra. Es necesario señalar aquí que el efecto Doppler observado en las galaxias es producido realmente por la expansión del espacio (figura 1), algo de lo que era consciente Lemaître pero no Hubble (Kragh, 2011), que produce un alejamiento de la fuente emisora de luz análogo al efecto Doppler en ondas sonoras. A causa de esta expansión, los objetos que emitieron la luz hace unos 14.000 millones de años se encuentran actualmente a 46.000 millones de ańos-luz, distancia conocida como radio del universo observable (Davis y Lineweaver, 2004). 

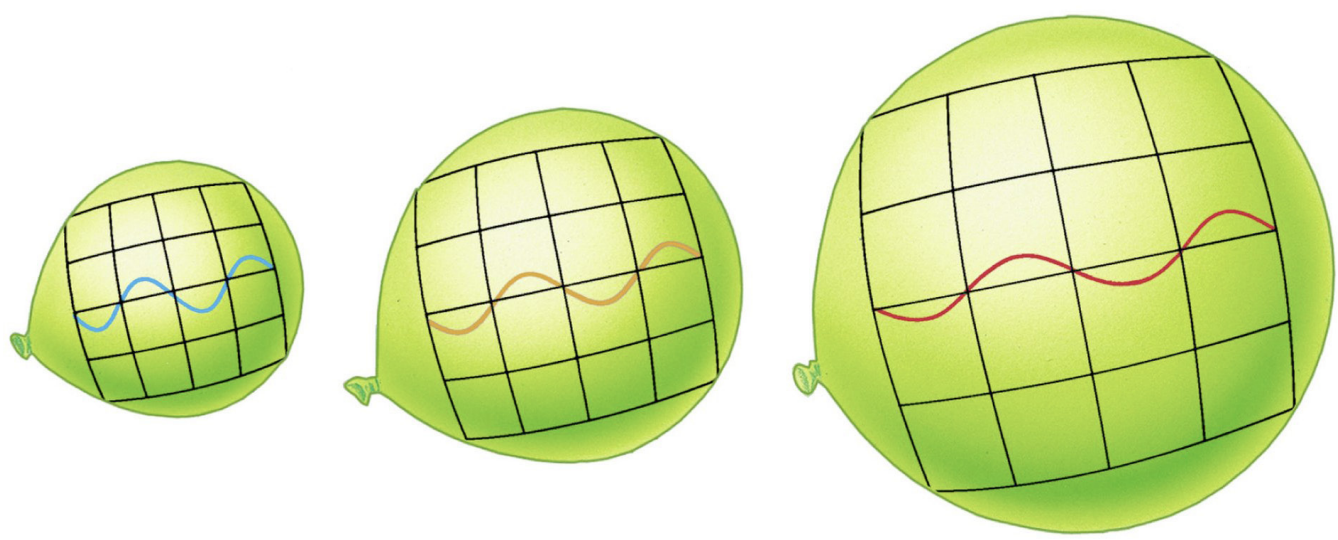

Fig. 1. Corrimiento al rojo de la luz causado por la expansión del universo (disponible en línea: <http://www2.astro.psu.edu/ users/cpalma/astro1h/class28.html>)

\section{METODOLOGÍA}

Inicialmente se realizó un ensayo piloto de la propuesta durante el curso 2010-2011. Una vez perfilada, se desarrolló la propuesta definitiva durante los cursos 2011-2012 y 2012-2013, en 13 sesiones, según el programa guía disponible en la página web <www.astrodidactica.com>, en al apartado «Propuesta CMC», e incluyendo además una salida extraescolar.

Con el fin de comprobar en qué medida la propuesta contribuyó a superar las dificultades de aprendizaje de los estudiantes, se utilizó el cuestionario ya descrito por Solbes y Palomar (2013), cuyos enunciados aparecen en la tabla 2. Cada uno de los doce ítems del cuestionario tenía categorías de respuesta distintas, seis de ellos $(2,4,5,7,8$ y 10) con categorías de correctas e incorrectas, y otros seis $(1,3,6$, 9,11 y 12) con categorías de respuestas correctas, parcialmente correctas e incorrectas. Estas categorías, sus porcentajes, y el enunciado de cada ítem pueden encontrarse en el apartado de resultados.

Para el tratamiento estadístico, realizado con el programa SPSS 20, las respuestas correctas puntuaron 2, las parcialmente correctas 1 y las incorrectas 0 . La validez y fiabilidad de este se contrastó mediante el uso de ítems utilizados en otras investigaciones y, posteriormente, con el cálculo del estadístico alfa de Cronbach (Cohen, Manion y Morrison, 2007) en uno de los grupos. El valor obtenido es $\alpha=0,711$, mayor que el aceptado en la bibliografía para indicar la consistencia interna del cuestionario.

Dicho cuestionario se aplicó previamente a la impartición de la unidad didáctica (pre-test) y posteriormente, después de pasar 7 días de la finalización de la propuesta (post-test). Con esta metodología se pretende comparar los resultados obtenidos en el post-test con los del pre-test, ítem a ítem, para ver si hay diferencias significativas entre estos. Esta comparación se realiza con la prueba estadística pertinente, según el tipo de muestra y variables, y se incluye el cálculo del tamaño del efecto, que estima la magnitud de la mejora y completa así la existencia de diferencias significativas.

El número de estudiantes que desarrollaron la propuesta definitiva fue de 99. Puesto que las CMC es una asignatura común y de carácter obligatorio para todos los estudiantes de bachillerato (17 años), en la muestra se encuentran 40 estudiantes de la modalidad científico-tecnológica $(40,4 \%)$ y 59 de la modalidad de humanidades-ciencias sociales $(59,6 \%)$.

La convivencia de estas dos modalidades de bachillerato podría generar diferencias en los resultados de la asignatura, puesto que los estudiantes de la modalidad de humanidades-ciencias sociales hace dos años ( $3 .^{\circ}$ de ESO, 15 años) que no estudian una asignatura de carácter científico. Así, este será uno de los aspectos que se deberán indagar en el análisis de los resultados. 


\section{PRESENTACIÓN Y ANÁLISIS DE LOS RESULTADOS}

\section{Resultados al comparar pre-test y post-test}

Inicialmente se realiza en este apartado el análisis estadístico comparando pre-test y post-test, sin atender a la influencia de la modalidad de bachillerato, que se abordará en un apartado siguiente. Antes de valorar los resultados pre-test y post-test en cada uno de los ítems, se realizan algunas pruebas estadísticas con el fin de comprobar si existen diferencias estadísticamente significativas en cada uno de los ítems del pre-test y el post-test. Puesto que se trata de muestras relacionadas (pre y post) que no siguen una distribución normal de forma simultánea (según prueba de Kolmogorov-Smirnov), se escogen pruebas de homogeneidad marginal.

Puesto que los ítems poseen distinto número de categorías, no se puede aplicar la misma prueba de contraste de homogeneidad marginal para todos. Para constatar la significación de los cambios en el caso de los ítems con dos categorías, se utiliza la prueba no paramétrica de McNemar, y para los ítems con tres categorías la prueba no paramétrica Stuart-Maxwell. La tabla 2 muestra los enunciados de los ítems y si hay diferencias significativas entre el pre y el post.

Tabla 2.

Significación estadística para cada ítem según la prueba

de homogeneidad marginal realizada y el tamaño del efecto calculado mediante la $\mathrm{d}$ de Cohen

\begin{tabular}{|c|c|c|c|c|}
\hline Ítem & Sig. asint. & $\begin{array}{c}\text { Diferencia } \\
\text { estadisticamente } \\
\text { significativa } \\
(a<0,05)\end{array}$ & $\begin{array}{c}d \\
\text { Cohen }\end{array}$ & $\begin{array}{c}\text { Efecto } \\
\text { Hattie (2009) }\end{array}$ \\
\hline 1. Explica cómo te orientarías de noche y de día. & 0,000 & sí & 0,64 & Grande \\
\hline $\begin{array}{l}\text { 2. Comenta la frase: «El sol sale por el este y se pone por el } \\
\text { oeste». }\end{array}$ & 0,000 & sí & 0,72 & Grande \\
\hline $\begin{array}{l}\text { 3. Señala aplicaciones que conozcas sobre la astronomía e } \\
\text { indica en qué observaciones se basan. }\end{array}$ & 0,000 & sí & 0,44 & Medio \\
\hline $\begin{array}{l}\text { 4. ¿Cómo podemos explicar astronómicamente las estacio- } \\
\text { nes del año? }\end{array}$ & 0,000 & sí & 0,73 & Grande \\
\hline 5. Explica las fases de la Luna. & 0,002 & sí & 0,33 & Medio \\
\hline $\begin{array}{l}\text { 6. ¿Qué hechos pusieron en cuestión el modelo geocéntri- } \\
\text { co? }\end{array}$ & 0,000 & sí & 0,65 & Grande \\
\hline $\begin{array}{l}\text { 7. Explica cómo la observación de la Vía Láctea demuestra } \\
\text { que estamos en una galaxia. }\end{array}$ & 0,000 & sí & 0,46 & Medio \\
\hline $\begin{array}{l}\text { 8. Si la distancia del Sol a Neptuno fuera como un campo } \\
\text { de fútbol }(110 \mathrm{~m}) \text {, ¿qué tamaño crees que tendría la Tie- } \\
\text { rra? ¿Y el Sol? ¿Y Júpiter? }\end{array}$ & 0,453 & no & 0,14 & Pequeño \\
\hline $\begin{array}{l}\text { 9. Cita tecnologías que han contribuido al desarrollo de la } \\
\text { astronomía. }\end{array}$ & 0,005 & sí & 0,35 & Medio \\
\hline $\begin{array}{l}\text { 10. Explica con tus propias palabras la expansión del univer- } \\
\text { so. }\end{array}$ & 0,000 & sí & 0,68 & Grande \\
\hline 11. ¿Qué pruebas hay de la expansión del universo? & 0,000 & sí & 0,65 & Grande \\
\hline $\begin{array}{l}\text { 12. Comenta la frase: «La astronomía y la astrología son cien- } \\
\text { cias distintas». }\end{array}$ & 0,000 & sí & 0,61 & Grande \\
\hline
\end{tabular}

En la tabla 2 se observan diferencias estadísticamente significativas en todos los ítems excepto en el 8 , y si se calcula el tamaño del efecto mediante la d de Cohen se obtienen 7 ítems con efecto grande (d 
$>0,60), 4$ con efecto medio $(0,30<\mathrm{d}<0,59)$ y 1 con pequeño $(\mathrm{d}<0,29)$ (Hattie, 2009). Además, si se tiene en cuenta la contribución de todos los ítems al resultado global del cuestionario, se obtiene un valor para el tamaño del efecto de 1,13, dentro del rango de efectos grandes considerados por Hattie (2009) y muy fuerte según algunos autores (Cohen, Manion y Morrison, 2007).

Estas diferencias se observan gráficamente en la figura 2, que muestra los resultados obtenidos en pre y post.

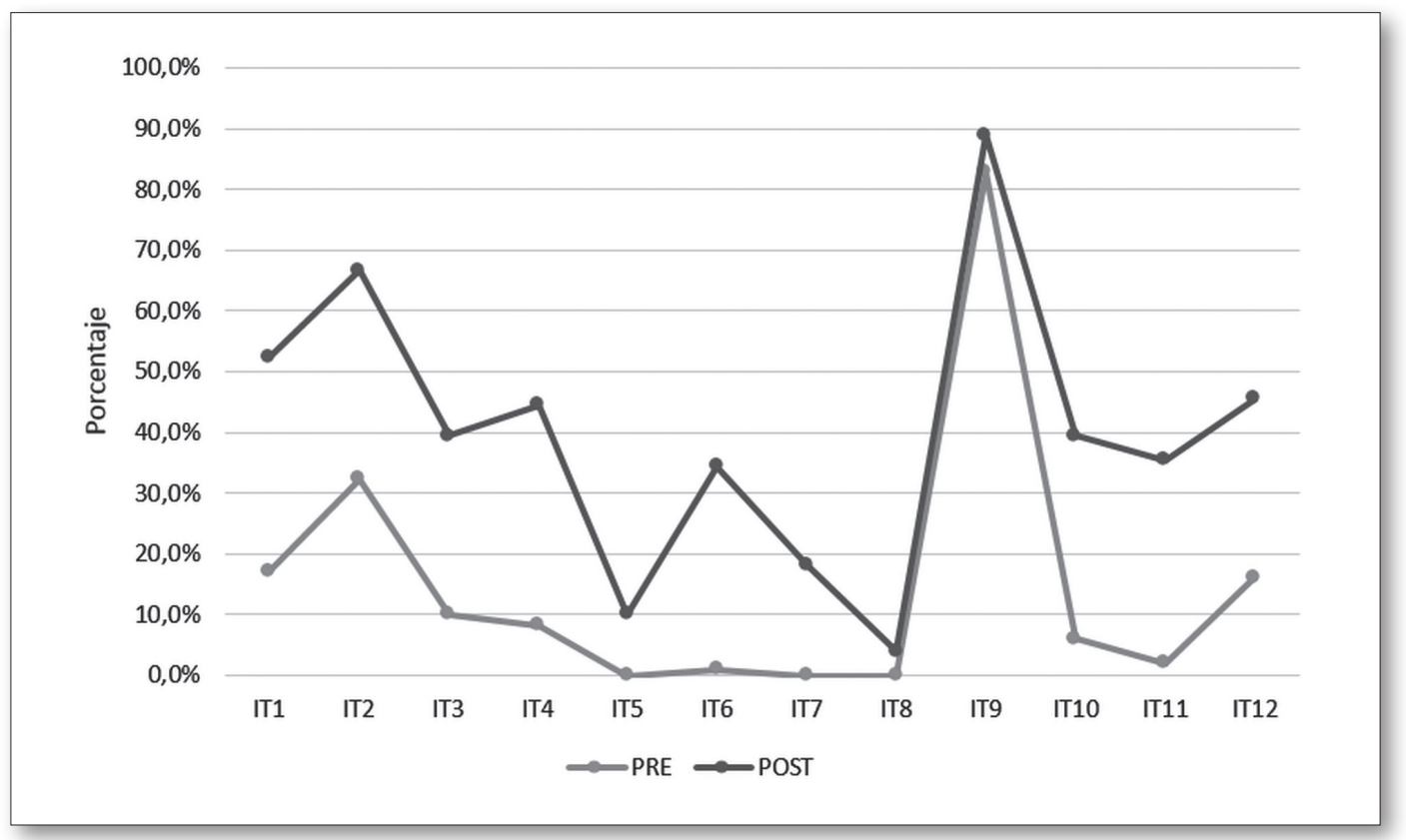

Fig. 2. Representación del porcentaje de la suma de respuestas correctas y parcialmente correctas de cada ítem.

Además de la comparación estadística reflejada en la tabla 2, en la figura 2 se han sumado el número de respuestas correctas (2) y parcialmente correctas (1), y se ha realizado el porcentaje sobre el total de alumnos, y puede observarse cómo, en líneas generales, los puntos discurren por el gráfico manteniendo la forma. Además, se aprecian sensibles diferencias entre el grupo pos-test y el pre-test.

A continuación se muestran las tablas correspondientes a cada ítem, en las que aparecen las categorías de respuestas, la puntuación para realizar el tratamiento estadístico y los porcentajes obtenidos en pre-test y post-test. Además, se describen estos porcentajes para cada ítem y se comenta qué ha sucedido en cada uno de ellos.

Tabla 3.

Respuestas al ítem 1: Explica cómo te orientarías de noche y de día

\begin{tabular}{|l|c|c|c|}
\hline \multicolumn{1}{|c|}{ Categoría } & Puntos & Pre & Post \\
\hline $\begin{array}{l}\text { Explican correctamente ambas situaciones, el día } \\
\text { y la noche }\end{array}$ & 2 & $7,1 \%$ & $22,2 \%$ \\
\hline $\begin{array}{l}\text { Explican correctamente una situación, bien de día, } \\
\text { bien de noche }\end{array}$ & 1 & $10,1 \%$ & $30,3 \%$ \\
\hline Respuestas incorrectas & 0 & $75,8 \%$ & $47,5 \%$ \\
\hline No contesta & 0 & $7,1 \%$ & $0 \%$ \\
\hline
\end{tabular}


Se observa la mejoría en las dos categorías de repuestas correctas, con respuestas que contienen distinto tipo de detalle, desde las menos numerosas que detallaban algún procedimiento, a las que identificaban las parejas Norte-Estrella Polar y Este-Orto solar. Esta mejora puede estar causada no solo por el aprendizaje por parte de los alumnos de una forma de orientación, sino por la intención, que impregna toda la propuesta, de mostrar la necesidad de la astronomía para la especie.

Tabla 4.

Respuestas al ítem 2: Comenta la frase:

El Sol sale por el Este y se pone por el Oeste

\begin{tabular}{|l|c|c|c|}
\hline \multicolumn{1}{|c|}{ Categoría } & Puntos & Pre & Post \\
\hline $\begin{array}{l}\text { Respuesta correcta haciendo referencia a la } \\
\text { rotación de la Tierra }\end{array}$ & 2 & $32,3 \%$ & $66,7 \%$ \\
\hline $\begin{array}{l}\text { Confusión explícitamente geocéntrica, in- } \\
\text { cluso repitiendo la misma frase }\end{array}$ & 0 & $32,3 \%$ & $16,2 \%$ \\
\hline Otras respuestas incorrectas & 0 & $16,2 \%$ & $11,1 \%$ \\
\hline No contesta & 0 & $19,2 \%$ & $6,1 \%$ \\
\hline
\end{tabular}

Las confusiones explícitamente geocéntricas dan cuenta de la fuerte relación existente entre la observación y la creación de un modelo, puesto que muchos mantienen la idea del movimiento del Sol alrededor de la Tierra pese a conocer el modelo heliocéntrico (principalmente por las representaciones existentes). Esto puede ser debido a que los alumnos tienen un SR absoluto (ellos mismos en la superficie de la Tierra) y no han pasado a SR relativos y, por eso, es importante unificar cuantas más observaciones mejor en un único modelo, y el modelo heliocéntrico es más satisfactorio en este sentido. La propuesta realizada se ha revelado como bastante eficaz, reduciendo las confusiones explícitas y aumentando las respuestas correctas. La categoría "No contesta» se ha visto reducida, lo que indica que al menos el alumnado ha tratado de encontrar ahora una explicación a esta afirmación coloquial.

Tabla 5.

Respuestas al ítem 3: Señala aplicaciones que conozcas

sobre la astronomía e indica en qué observaciones se basan

\begin{tabular}{|l|c|c|c|}
\hline \multicolumn{1}{|c|}{ Categoría } & Puntos & Pre & Post \\
\hline $\begin{array}{l}\text { Citan aplicaciones indicando la observación } \\
\text { en que se basan }\end{array}$ & 2 & $3,0 \%$ & $10,1 \%$ \\
\hline Citan aplicaciones sin indicar observaciones & 1 & $7,1 \%$ & $29,3 \%$ \\
\hline Respuestas incorrectas & 0 & $22,2 \%$ & $16,2 \%$ \\
\hline No contesta & 0 & $67,7 \%$ & $44,4 \%$ \\
\hline
\end{tabular}

Esta mejora en el porcentaje de estudiantes que contestaron total o parcialmente a la pregunta puede ser consecuencia, como ya se señalaba en el ítem 1, de la insistencia de la propuesta en mostrar las aplicaciones de la astronomía, sobre todo en los comienzos de la humanidad, evitando presentarla como una simple acumulación de conceptos abstractos. Un análisis más detallado de las respuestas correctas confirma esta interpretación de la mejora, pues el incremento de respuestas correctas se ha producido sobre todo en las que podrían denominarse aplicaciones clásicas de la astronomía (calendario, agricultura y orientación), mientras otras aplicaciones astronómicas más modernas (satélites, meteorología, mareas) se mantienen igual. 
Tabla 6.

Respuestas al ítem 4: ¿Cómo podemos

explicar astronómicamente las estaciones del año?

\begin{tabular}{|l|c|c|c|}
\hline \multicolumn{1}{|c|}{ Categoría } & Puntos & Pre & Post \\
\hline $\begin{array}{l}\text { Explican astronómicamente las estaciones } \\
\text { nombrando la inclinación del eje }\end{array}$ & 2 & $7,1 \%$ & $42,4 \%$ \\
\hline $\begin{array}{l}\text { Describen cómo determinar astronómica- } \\
\text { mente mediante la observación de las cons- } \\
\text { telaciones estacionales }\end{array}$ & 2 & $1,0 \%$ & $2,0 \%$ \\
\hline Respuestas incorrectas & 0 & $64,6 \%$ & $53,5 \%$ \\
\hline No contesta & 0 & $27,3 \%$ & $2,0 \%$ \\
\hline
\end{tabular}

La propuesta de enseñanza incluía actividades destinadas a recordar de su formación anterior que la inclinación de los rayos del Sol (consecuencia de la inclinación del eje terrestre) es la causante del aumento de la temperatura y por tanto de las estaciones. Puede atribuirse la mejora a la realización de estas actividades. La insistencia en el hecho de que la órbita de la Tierra es prácticamente circular y la aparente contradicción para el estudiante de que en el hemisferio norte es verano cuando la Tierra está más alejada del Sol contribuyen a reforzar este conocimiento. Esto último puede intuirse al observar que mientras en el pre-test hubo 3 estudiantes que señalaron de alguna forma la diferencia entre hemisferios, en el post-test fueron 9 los que dieron cuenta de este detalle. Aunque muy minoritarias, también existen contestaciones que van dirigidas a explicar la estación presente mediante las constelaciones estacionales visibles en ese momento, pasando de un 1 a un $2 \%$.

Tabla 7.

Respuestas al ítem 5: Explica las fases de la Luna

\begin{tabular}{|l|c|c|c|}
\hline \multicolumn{1}{|c|}{ Categoría } & Puntos & Pre & Post \\
\hline $\begin{array}{l}\text { Explica las fases de la Luna correctamente in- } \\
\text { dicando la posición relativa del sistema Tierra- } \\
\text { Sol-Luna }\end{array}$ & 2 & $0,0 \%$ & $10,1 \%$ \\
\hline Respuestas incorrectas & 0 & $78,8 \%$ & $77,8 \%$ \\
\hline No contesta & 0 & $21,2 \%$ & $12,1 \%$ \\
\hline
\end{tabular}

Este ítem no posee ninguna respuesta correcta en el pre-test, pudiendo encontrar la causa en que bien la pregunta no ha sido entendida o bien el alumnado olvida rápidamente la relación entre las posiciones Sol-Tierra-Luna y las fases de esta última. El estudio de las fases lunares se realiza en la educación primaria (6-12 años) y en el primer curso de la educación secundaria (13 años), y está abordado en la bibliografía desde diferentes ángulos (Trundle, Atwood y Chistopher, 2007; Vega, 2001; Mulholland y Ginns, 2008; Ogan-Bekiroglu, 2007; Trumper, 2001). Después de aplicar la propuesta, los resultados del post-test son mejores. Esto sucede a pesar de la complejidad del problema, dado que supone relacionar las observaciones de la Luna realizadas desde la superficie de la Tierra con el diagrama de la Luna alrededor de la Tierra iluminadas por el Sol (y vistas por un observador situado sobre el plano Tierra, Luna y Sol), lo que no es nada sencillo. 
Tabla 8 .

Respuestas al ítem 6:

Qué hechos pusieron en cuestión el modelo geocéntrico

\begin{tabular}{|l|c|c|c|}
\hline \multicolumn{1}{|c|}{ Categoría } & Puntos & Pre & Post \\
\hline Contesta con dos hechos o más & 2 & $0,0 \%$ & $5,1 \%$ \\
\hline Contesta con un hecho & 1 & $1,0 \%$ & $29,3 \%$ \\
\hline Respuestas incorrectas & 0 & $37,4 \%$ & $36,4 \%$ \\
\hline No contesta & 0 & $61,6 \%$ & $29,3 \%$ \\
\hline
\end{tabular}

Los hechos más comunes son la observación de los satélites de Júpiter, del movimiento de retrogradación de los planetas y de las fases de Venus. La mejora en las dos categorías de respuestas correctas se debe principalmente a las respuestas que hacen referencia a la no concordancia de la órbita de algunos planetas con el modelo de Ptolomeo, señalada por un 26,3\% de estudiantes. El descubrimiento de los satélites de Júpiter, con un 11,1\% de los estudiantes, y las fases de Venus, con un 5,1\%, son los otros hechos señalados. Estos tres últimos porcentajes no suman el porcentaje total de respuestas correctas puesto que las categorías no son disjuntas.

La diferencia de respuestas correctas en pre-test y post-test es una de las mayores (véase figura 2). Este hecho puede ser debido a que tanto en cursos anteriores, como en un tratamiento habitual, en el mejor de los casos no se insiste en la explicación de las observaciones a partir de los modelos cosmológicos previos al actual, y en el peor no se realiza un tratamiento histórico. La propuesta realizada busca ambos objetivos, un acercamiento histórico que revele las dificultades que surgieron y una explicación de las observaciones a partir de un determinado modelo.

Tabla 9.

Respuestas al ítem 7: Explica cómo la observación de la Via Láctea demuestra que estamos en una galaxia

\begin{tabular}{|l|c|c|c|}
\hline \multicolumn{1}{|c|}{ Categoría } & Puntos & Pre & Post \\
\hline $\begin{array}{l}\text { Agrupación de estrellas en el plano de la } \\
\text { galaxia }\end{array}$ & 2 & $0,0 \%$ & $18,2 \%$ \\
\hline Respuestas incorrectas & 0 & $23,2 \%$ & $56,6 \%$ \\
\hline No contesta & 0 & $76,8 \%$ & $25,3 \%$ \\
\hline
\end{tabular}

De nuevo se produce una ausencia de respuestas correctas en el pre-test en una pregunta relacionada con la observación, fruto tal vez de una enseñanza de la astronomía demasiado teórica a pesar de tratarse de una disciplina muy observacional. La mejora debida a la propuesta puede tener su origen en la insistencia en explicar los modelos a partir de las observaciones que ya se ha citado anteriormente.

Tabla 10.

Respuestas al ítem 8: Si la distancia del Sol a Neptuno fuera como un campo de fútbol $(110 \mathrm{~m})$, ¿qué tamaño crees que tendria la Tierra? ¿Y el Sol? ¿Y Júpiter?

\begin{tabular}{|l|c|r|r|}
\hline \multicolumn{1}{|c|}{ Categoría } & Puntos & \multicolumn{1}{c|}{ Pre } & \multicolumn{1}{c|}{ Post } \\
\hline La respuesta guarda las proporciones adecuadas & 2 & $2,0 \%$ & $5,0 \%$ \\
\hline Respuestas incorrectas & 0 & $20,2 \%$ & $34,3 \%$ \\
\hline No contesta & 0 & $77,8 \%$ & $60,6 \%$ \\
\hline
\end{tabular}


La respuesta correcta consiste en conocer y aplicar las proporciones adecuadas de las magnitudes de los cuerpos (diámetro de Júpiter 10 veces el de la Tierra y diámetro del Sol 10 veces el de Júpiter). Esa respuesta tan solo la han conseguido un $5 \%$ en post-test y, de estos, solo el 1\% acierta además con el orden de magnitud de esta proporción. Estos resultados, que muestran una mejora no significativa (tabla 2), ponen de manifiesto la dificultad señalada (tabla 1) y sugieren la necesidad de una mayor insistencia de las actividades de escala en tratamientos futuros. Realizar una actividad en la que aparezcan correctamente las escalas de diámetros y distancias en el sistema solar no es fácil pero tampoco imposible. En la propuesta sí se ha planteado una actividad de este estilo, y ello puede llevar a pensar que quizá la pregunta planteada en el cuestionario era exigente para resolverla sin datos. Las respuestas incorrectas pasaron de un 20,2 a un 34,3\%, y los estudiantes que no contestaron a la pregunta disminuyen de un 77,8 a un 60,6\%, lo que indica, como en el resto de ítems, que los estudiantes han tratado de contestar más en el post-test que en el pre-test, aunque no hayan tenido éxito.

Tabla 11.

Respuestas al ítem 9: Cita tecnologías

que han contribuido al desarrollo de la astronomía

\begin{tabular}{|l|c|c|c|}
\hline \multicolumn{1}{|c|}{ Categoría } & Puntos & \multicolumn{1}{c|}{ Pre } & \multicolumn{1}{c|}{ Post } \\
\hline Cita al menos dos tecnologías & 2 & $31,3 \%$ & $49,5 \%$ \\
\hline Cita una tecnología & 1 & $51,5 \%$ & $39,4 \%$ \\
\hline Respuestas incorrectas & 0 & $6,1 \%$ & $4,0 \%$ \\
\hline No contesta & 0 & $11,1 \%$ & $7,1 \%$ \\
\hline
\end{tabular}

Se esperaban respuestas del tipo: telescopios, radiotelescopios, satélites, gnomon, astrolabio, etc. Este ítem es el que mejor resultados obtuvo en el pre-test, puesto que existe una tecnología ampliamente ligada a la astronomía como es el telescopio. Pese a producirse una mejora estadísticamente significativa, el tamaño del efecto es medio (tabla 2) dada la dificultad que implica mejorar algo ya de por sí alto. Vale la pena señalar aquí que aparecen otros instrumentos que en el pre-test no figuraban, nombrados por un $16,2 \%$ de los estudiantes, como el gnomon, el reloj de sol, el astrolabio o el espectroscopio. La aparición de estos instrumentos puede ser debida al recorrido histórico que ha servido de hilo conductor de la propuesta.

Tabla 12.

Respuestas al ítem 10: Explica con tus propias palabras la expansión del universo

\begin{tabular}{|l|c|c|c|}
\hline \multicolumn{1}{|c|}{ Categoría } & Puntos & \multicolumn{1}{c|}{ Pre } & Post \\
\hline Explicación de la expansión a raíz del Big Bang & 2 & $6,1 \%$ & $39,4 \%$ \\
\hline Respuestas incorrectas & 0 & $32,3 \%$ & $45,5 \%$ \\
\hline No contesta & 0 & $62 \%$ & $15,2 \%$ \\
\hline
\end{tabular}

Un 39,4\% de los estudiantes en el post-test han sido capaces de señalar de una forma u otra que la explosión inicial, conocida como Big Bang, es la que causa la expansión del universo, frente al 6,1\% que lo hizo en pre-test. Alguno de ellos va un poco más allá y nombra cómo el tiempo y el espacio se crean en el momento de la explosión, de manera que las preguntas sobre qué había antes y dónde se produjo dejan de tener sentido. Por otra parte, se ha producido una disminución de casi el $50 \%$ en el abandono de la pregunta, lo que indica que el estudiante acaba el tema con la idea de que es capaz de contestar correctamente aunque parte de ellos lo hagan mal, tal como se ha visto en el aumento de respuestas incorrectas. 
Tabla 13.

Respuestas al ítem 11: Qué pruebas hay de la expansión del universo

\begin{tabular}{|l|c|c|c|}
\hline \multicolumn{1}{|c|}{ Categoría } & Puntos & Pre & \multicolumn{1}{c|}{ Post } \\
\hline Cita tres pruebas de la expansión & 2 & $0,0 \%$ & $4,0 \%$ \\
\hline Cita dos pruebas de la expansión & 2 & $0,0 \%$ & $5,1 \%$ \\
\hline Cita una prueba de la expansión & 1 & $2,0 \%$ & $26,3 \%$ \\
\hline Respuestas incorrectas & 0 & $16,2 \%$ & $39,4 \%$ \\
\hline No contesta & 0 & $81,8 \%$ & $25,3 \%$ \\
\hline
\end{tabular}

Las tres pruebas más habituales son la radiación de fondo, el desplazamiento al rojo y la abundancia de núcleos ligeros. La gran mejora producida se debe a la prueba que más estudiantes han nombrado en sus respuestas: el alejamiento de las galaxias $(33,3 \%)$. La radiación de fondo, con un 10,1\% de los estudiantes, y la abundancia de elementos ligeros, con un 5,1\% (estas categorías no son disjuntas puesto que existen respuestas con más de una prueba).

La insistencia en la necesidad de probar los enunciados en toda la propuesta, junto con la aproximación histórica al nacimiento de la teoría del Big Bang, promueven este aumento de respuestas correctas, centradas en la prueba del desplazamiento al rojo, puesto que es la más fácil de entender por los y las estudiantes de este nivel. Además, este ítem ha experimentado la mayor disminución en el porcentaje de estudiantes que dejaron la pregunta sin contestar (81,8 a 25,3\%), síntoma que puede deberse a un acercamiento a la teoría del Big Bang, antes posiblemente conocida solo por el nombre.

Tabla 14.

Respuestas al ítem 12: Comenta la frase:

La astronomía y la astrología son ciencias distintas

\begin{tabular}{|l|c|c|c|}
\hline \multicolumn{1}{|c|}{ Categoría } & Puntos & Pre & Post \\
\hline Diferencia y señala que la astrología no es una ciencia & 2 & $7,1 \%$ & $33,3 \%$ \\
\hline $\begin{array}{l}\text { Diferencia pero no señala que la astrología no es una } \\
\text { ciencia }\end{array}$ & 1 & $9,1 \%$ & $12,1 \%$ \\
\hline Respuestas incorrectas & 0 & $36,4 \%$ & $31,3 \%$ \\
\hline No contesta & 0 & $47,5 \%$ & $23,2 \%$ \\
\hline
\end{tabular}

Las respuestas correctas, aquellas que señalan el carácter acientífico de la astrología, aumentan de un 7,1 al 33,3\%, con palabras del tipo creencia, pseudociencia o ilusión. La propuesta trata de eliminar la inercia astrológica que los estudiantes arrastran, en parte por culpa de los medios de comunicación, y fomentar el espíritu crítico. Esto se consigue planteando alguna actividad que sorprende al estudiante por su final inesperado (véanse actividades ejemplo). Existe otro tipo de respuesta en la que el estudiante ha diferenciado ambas disciplinas, pero no ha sido capaz de señalar el carácter acientífico de la astrología, considerándose respuestas parcialmente correctas, y que han aumentado a un $12,1 \%$ en el post-test.

Las dos cuestiones anteriores (ítems 11 y 12) refuerzan la necesidad de probar los enunciados científicos y contribuyen a la formación del pensamiento crítico del estudiante. Es precisamente esta forma de pensar la que consigue que el estudiante rechace la astrología como una ciencia, como refleja el aumento en el porcentaje del alumnado que realiza esta distinción. 


\section{Influencia de la modalidad escogida por el estudiante}

Para valorar la influencia de la modalidad inicialmente se pensó en utilizar una prueba ANOVA $2 \times 2$ mixta, pero al no cumplirse la igualdad de varianzas, no pudo realizarse. El análisis escogido consiste en comparar la significación estadística, mediante el estadístico correspondiente, de cada ítem de los siguientes subgrupos:

- Pre-test de humanidades-ciencias sociales frente a pre-test de científico-tecnológico.

- Pre-test frente a post-test de científico-tecnológico.

- Pre-test frente a post-test de humanidades-ciencias sociales.

- Post-test de humanidades-ciencias sociales frente a post-test de científico-tecnológico.

Los resultados obtenidos son los siguientes:

Pre-test de humanidades-ciencias sociales frente a pre-test de científico-tecnológico

Para ver si existen diferencias significativas en las contestaciones realizadas en cada uno de los ítems del pre-test según sea la modalidad del estudiante, se utiliza el estadístico Chi-cuadrado de Pearson, puesto que se trata de muestras independientes que no siguen una distribución normal de forma simultánea (según test de Kolmogorov-Smirnov). Los valores obtenidos para la significación asintótica aparecen en la tabla 15.

Tabla 15.

Significación estadística obtenida mediante

Chi-cuadrado al comparar el pre-test de ambas modalidades

\begin{tabular}{|l|c|c|c|c|c|c|c|c|c|c|c|c|}
\hline & ÍT1 & ÍT2 & ÍT3 & ÍT4 & ÍT5 & ÍT6 & ÍT7 & ÍT8 & ÍT9 & ÍT10 & ÍT11 & ÍT12 \\
\hline Significación &, 234 &, 492 &, 531 &, 341 & - &, 844 & - &, 314 &, 000 & 1 & 1 &, 609 \\
\hline
\end{tabular}

En los ítems 5 y 7 no se ha podido calcular el estadístico puesto que los resultados son iguales en ambos subgrupos. La primera conclusión que se extrae al analizar estos datos es que en casi la totalidad de ítems no existen diferencias significativas, excepto en el ítem 9, en el que el resultado sí es menor que 0,05 y por tanto las diferencias son significativas. Es decir, los estudiantes de ambas modalidades empiezan con conocimientos similares sobre astronomía. La segunda conclusión que puede extraerse es que la formación astronómica recibida previamente por los alumnos ha tenido poco calado: los alumnos de la modalidad de humanidades-ciencias sociales no reciben contenidos astronómicos desde hace 4 cursos y pueden entenderse estos resultados, pero lo sorprendente es que los alumnos de la modalidad científico-tecnológica han estudiado el curso anterior el bloque de fuerzas y movimientos en el que se tratan muchos de los conceptos de nuestra propuesta. Esta falta de conocimiento sobre astronomía por parte del alumnado científico-tecnológico puede estar causada por un tratamiento más superficial, meramente informativo y cuantitativo, que lo aparta de un tratamiento más indagativo, histórico y observacional, que es el que aquí se propone. La diferencia significativa entre los alumnos de las dos modalidades que aparece en el ítem 9, el que relaciona la tecnología con la astronomía, se debe a que los alumnos de la científico-tecnológica son capaces de reconocer más fácilmente las relaciones entre ciencia y tecnología antes del pre-test. 
Pre-test frente a post-test de científico-tecnológico

Los mismos test de homogeneidad marginal realizados para toda la muestra pueden realizarse para el subgrupo de la modalidad del científico-tecnológico. Los resultados aparecen en la tabla 16 y en la figura 3.

Tabla 16.

Significación estadística obtenida mediante test de homogeneidad marginal al comparar el pre y post de la modalidad científico-tecnológica

\begin{tabular}{|l|c|c|c|c|c|c|c|c|c|c|c|c|}
\hline & ÍT1 & ÍT2 & ÍT3 & ÍT4 & ÍT5 & ÍT6 & ÍT7 & ÍT8 & ÍT9 & ÍT10 & ÍT11 & ÍT12 \\
\hline Significación &, 000 &, 000 &, 000 &, 001 &, 008 &, 000 &, 000 &, 453 &, 020 &, 000 &, 000 &, 000 \\
\hline
\end{tabular}

Casi la totalidad de los ítems obtienen significación menor de 0,05, lo que indica que existen mejoras estadísticamente significativas en esos ítems para el subgrupo de la modalidad científico-tecnológica. Es el ítem 8 el que vuelve a no obtener mejora, tal y como se comentó anteriormente, a pesar de considerar solo el subgrupo de ciencias. Esto nos indica que la propuesta ha producido mejoras significativas en el aprendizaje de los estudiantes de la modalidad científico-tecnológica.

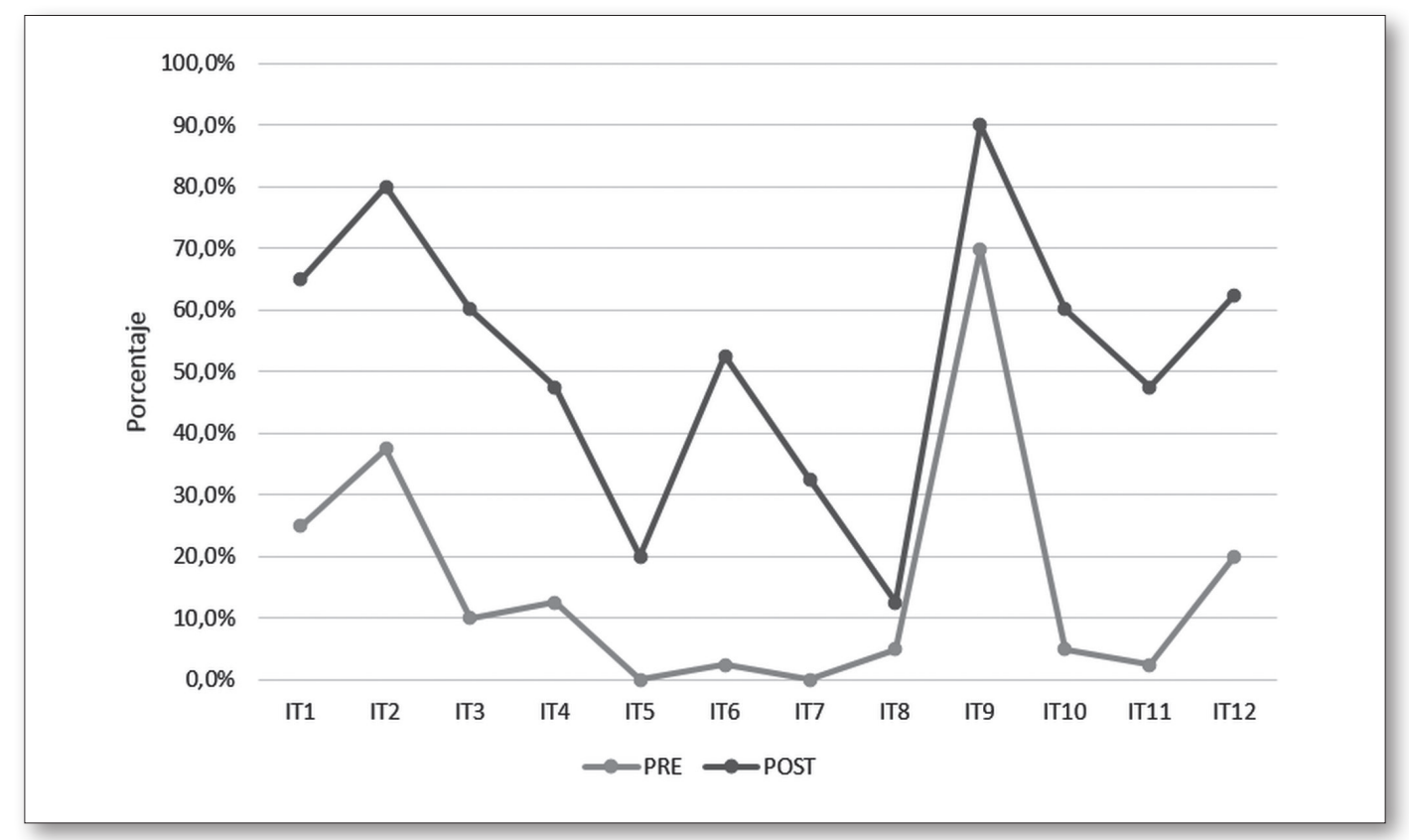

Fig. 3. Gráfica que representa los porcentajes de los 40 estudiantes de científico-tecnológico que contestan correctamente cada ítem.

Pre-test frente a post-test de humanidades-ciencias sociales

De igual modo, los resultados de los test de homogeneidad marginal para el subgrupo de humanidades-ciencias sociales aparecen en la tabla 17 y en la figura 4. 
Tabla 17.

Significación estadística obtenida mediante test de homogeneidad marginal al comparar el pre y post de la modalidad humanidades-ciencias sociales

\begin{tabular}{|l|c|c|c|c|c|c|c|c|c|c|c|c|}
\hline & ÍT1 & ÍT2 & ÍT3 & ÍT4 & ÍT5 & ÍT6 & ÍT7 & ÍT8 & ÍT9 & ÍT10 & ÍT11 & ÍT12 \\
\hline Significación &, 000 &, 000 &, 739 &, 000 &, 500 &, 000 &, 063 & - &, 096 &, 003 &, 000 &, 000 \\
\hline
\end{tabular}

En este subgrupo la mejora estadísticamente significativa tiene lugar en menos ítems que en el subgrupo científico-tecnológico, aunque son más de la mitad en los que la significación es menor de 0,05 y por tanto existe mejora estadísticamente significativa. En el ítem 8 no puede calcularse el test puesto que no se obtuvo ninguna respuesta correcta en pre-test ni en post-test. La explicación a la falta de mejora en algunas cuestiones puede encontrarse en la diferencia entre ambos subgrupos. En efecto, el alumnado de la modalidad científico-tecnológica incorpora mejor los conocimientos de astronomía por la posible contribución de otros conocimientos científicos que el alumnado científico-tecnológico cursa, al afianzamiento de los nuevos, ya que la base cognitiva no se construye como un edificio, ladrillo a ladrillo, sino como una red o telaraña en las cuales un nuevo ítem se afianza mejor cuantos más enlaces existan y pueda establecer con otros nodos de la red, enriqueciendo el telar completo al fortalecer otros nodos, así sean distantes (Díaz, 2007).

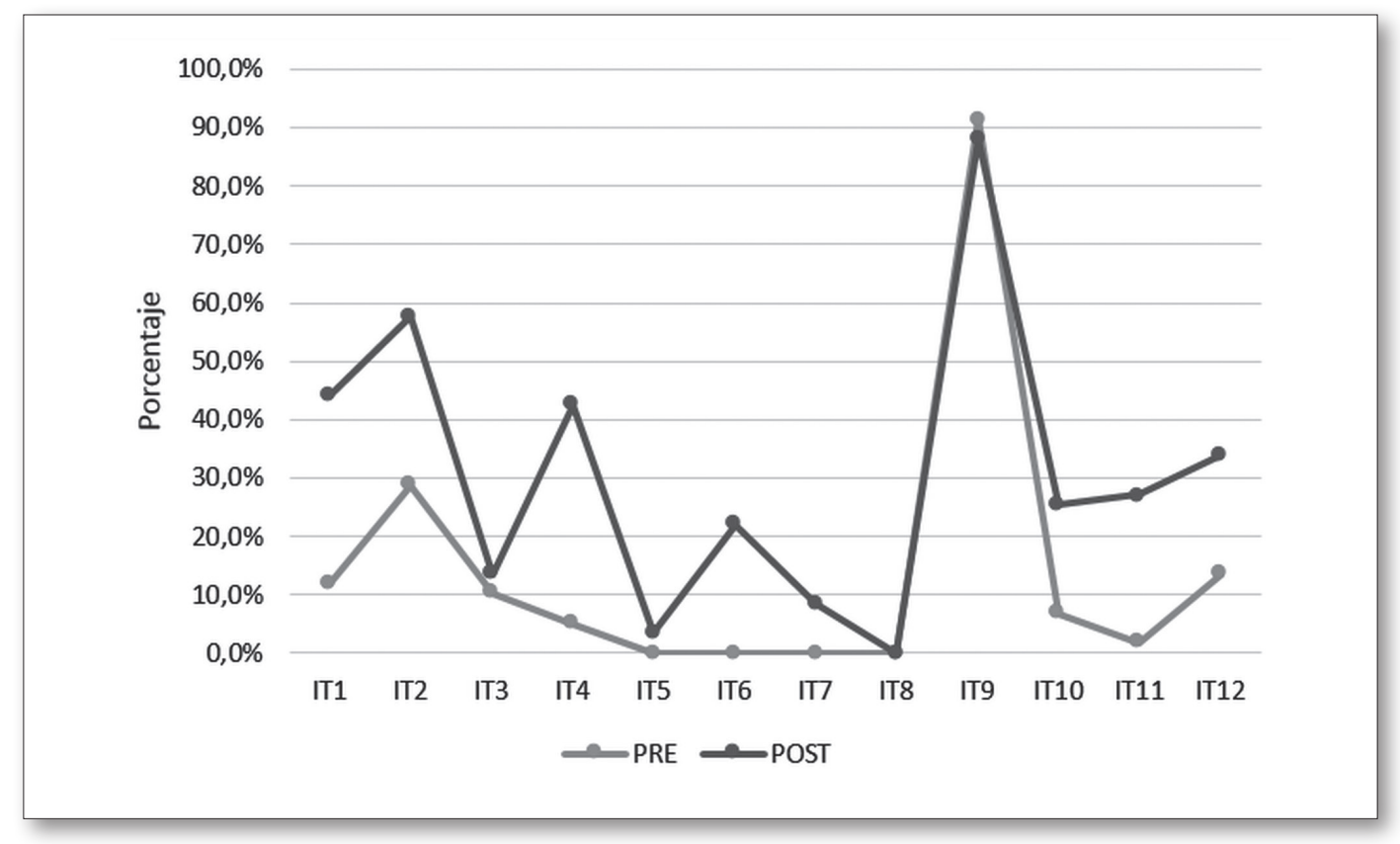

Fig. 4. Gráfica que representa los porcentajes de los 59 estudiantes de humanidades-ciencias sociales que contestan correctamente cada ítem.

Post-test de humanidades-ciencias sociales frente a post-test de científico-tecnológico

Puesto que se vuelven a comparar muestras independientes, se utiliza el estadístico Chi-cuadrado, con el que se obtienen los resultados que aparecen en la tabla 18. 
Tabla 18.

Significación estadística obtenida mediante

Chi-cuadrado al comparar el post-test de ambas modalidades

\begin{tabular}{|l|c|c|c|c|c|c|c|c|c|c|c|c|}
\hline & ÍT1 & ÍT2 & ÍT3 & ÍT4 & ÍT5 & ÍT6 & ÍT7 & ÍT8 & ÍT9 & ÍT10 & ÍT11 & ÍT12 \\
\hline Significación &, 064 &, 036 &, 000 &, 766 &, 019 &, 001 &, 006 &, 020 &, 215 &, 001 &, 016 &, 010 \\
\hline
\end{tabular}

Se observa cómo son minoría los ítems (1, 4 y 9) con significación menor de 0,05 y, por tanto, sin diferencias significativas. En el resto de ítems se puede afirmar que existen diferencias significativas entre los subgrupos de la modalidad en el post-test. Vale la pena señalar que el ítem 8, que no obtuvo mejora significativa al considerar el total del grupo, sí la obtiene aquí, lo que muestra que los estudiantes de la modalidad científico-tecnológica están más entrenados para estimar escalas y órdenes de magnitud.

Con esas cuatro pruebas realizadas, se demuestra que la propuesta ha sido más beneficiosa para los estudiantes de la modalidad científico-tecnológica. Se constata así la importancia del aprendizaje simultáneo en el curso actual de otras materias científicas y del interés de ese alumnado. Cabe suponer, además, que los alumnos de la modalidad científico-tecnológica están en principio más entrenados en realizar observaciones, asociarlas con modelos, reconocer las relaciones CTS, reforzar las teorías con pruebas, etc., cuestiones todas ellas tratadas en el tema diseñado.

\section{CONCLUSIONES Y PERSPECTIVAS}

Esta unidad didáctica ha sido utilizada con estudiantes de $1 .^{\circ}$ de bachillerato (16 años) y nos ha permitido comprobar que permite superar las dificultades de aprendizaje mostradas en Solbes y Palomar (2013). La propuesta facilita que los estudiantes se asomen a aspectos fundamentales de la actividad científica usualmente ignorados en la enseńanza como familiarizarse con la naturaleza de la ciencia, cuestionar las concepciones del universo y mostrar las relaciones CTS, todos ellos aspectos necesarios para romper con el creciente desinterés hacia los estudios de física señalado al principio de este trabajo.

La comprobación ha sido realizada al mostrar la significación estadística obtenida mediante las pruebas de homogeneidad marginal, el tamańo del efecto calculado mediante la d de Cohen y, finalmente, comparando los porcentajes de las categorías para valorar la magnitud de las diferencias en cada ítem.

La propuesta ha funcionado, dado que se han obtenido resultados estadísticamente significativos para todos los ítems, con tamaño de efecto grande en su mayoría (7), medio en 4 y pequeño en 1, el ítem 8, que guarda relación con la interpretación de las escalas en el sistema solar. Este ítem 8 requerirá en futuros tratamientos una atención especial, dada la dificultad que entraña manejar distancias y tiempos muy superiores a la escala humana. Si se considera el tamaño del efecto generado por la contribución de todos los ítems en conjunto, el resultado es un efecto más grande $(\mathrm{d}=1,13)$.

Mediante las pruebas estadísticas pertinentes se ha comprobado cómo los estudiantes de ambas modalidades comienzan el tema con conocimientos astronómicos similares, pese a que los estudiantes de la modalidad científico-tecnológica cursaron el año anterior contenidos de astronomía. Después de constatar la mejora estadísticamente significativa en ambas modalidades, se observa que los estudiantes de la modalidad científico-tecnológica han obtenido una mejora mayor que los de la modalidad de humanidades-ciencias sociales. En estos últimos, no ha habido mejora en los ítems relacionados con aspectos como las relaciones CTS, el cambio de sistema de referencia del observador y las escalas, conceptos en los que parecen estar más entrenados los estudiantes de la modalidad científico-tecnológica. 
En cuanto a las perspectivas, aunque las Ciencias para el Mundo Contemporáneo hayan sido lamentablemente suprimidas por la LOMCE, las actividades de la propuesta se pueden utilizar en cursos de secundaria en los que se imparta astronomía, en especial a partir de 4. ${ }^{\circ}$ de ESO.

\section{BIBLIOGRAFÍA}

Arribas, A. (2001). Astronomía paso a paso. Madrid: Equipo Sirius.

Atwood, R. у Aтwood, V. (1997). Effects of instruction on preservice elementary teacher's conceptions of the causes of night and day and the seasons. Journal of Science Teacher education, 8(1), pp. $1-13$.

http://dx.doi.org/10.1023/A:1009455201314

Camino, N. (1995). Ideas previas y cambio conceptual en astronomía. Un estudio con maestros de primaria sobre el día y la noche, las estaciones y las fases de la luna. Enseñanza de la ciencias, 13(1), pp. 81-96.

Cardenete, S. (2009). El sistema solar no nos cabe en el techo. Alambique 61, pp. 38-47.

Causeret, P., Fouquet, J. y Sarrazin-Vilas, L. (2008). El cielo al alcance de la mano. Madrid: Libsa.

Cohen, L., Manion, L. y Morrison, K. (2007). Research Methods in Education (6. a ed.). New York: Routledge.

Comins, N. F. (1993). Sources on Misconceptions in Astronomy. Third International Seminar on Misconceptions and Educational strategies in Science and Mathematics. New York, Cornell University: Ithaca.

Davis, T. y Lineweaver, C. (2004). Expanding confusion: Common misconceptions of cosmological horizons and the superluminical expansion of the universe. Astronomical Society of Australia, 21(1), pp. 97-109.

http://dx.doi.org/10.1071/AS03040

DíAz, J. (2007). La conciencia viviente. México D.F.: Fondo de Cultura Económica.

Dove, J. (2002). Does the man in the moon ever sleep? An analysis of students answers about simple astronomical events: a case study. International Journal of Science Education, 24(8), pp. 823-834. http://dx.doi.org/10.1080/09500690110066935

Forer, B. R. (1949). The fallacy of personal validation: A classroom demonstration of gullibility. Journal of Abnormal and Social Psychology, 44, pp. 118-123.

http://dx.doi.org/10.1037/h0059240

Grup Astre (1998). Materials didàctics per a l'ensenyament de l'Astronomia. Valencia: Nau Llibres. Premi Baldiri Reixach 1997.

Hansson, L. R. (2006). Swedish Upper Secondary Student's Views of the Origin and Development of the Universe. Research in Science Educations, 36, pp. 355-379. http://dx.doi.org/10.1007/s11165-005-9009-y

Hattie, J. (2009). Visible learning: A synthesis of meta-analyses in education. London: Routledge.

Jiménez Liso, M., López-GaY, R. y Martínez Chico, M. (2012). Cómo trabajar en el aula los criterios para aceptar o rechazar modelos científicos. Alambique 72, pp. 47-54.

Kragh, H. (2011). On Modern Cosmology and its Place in Science Education. Science \& Education, 20(3-4), pp. 343-357.

http://dx.doi.org/10.1007/s11191-010-9271-x

Lima, P. J., Lang Da Silveira, F. y Ostermann, F. (2012). Revista Brasileira de Ensino de Fisica, 34(1403). 
Mulholland, J. y Ginns, I. (2008). College Moon Project Austrlia: Preservice Teachers Learning about the Moon's Phases. Research in Science Education (38), pp. 385-399. http://dx.doi.org/10.1007/s11165-007-9055-8

Ogan-Bekiroglu, F. (2007). Effects of Model-based Teaching on Pre-service Physics Teachers' Conceptions of the Moon, Moon Phases, and Other Lunar Phenomena. International Journal of Science Education, 29(5), pp. 555-593. http://dx.doi.org/10.1080/09500690600718104

Palomar, R. (2013). Enseñanza y Aprendizaje de la astronomía en el bachillerato. Valencia: Universidad de Valencia. Disponible en línea: <http://roderic.uv.es/handle/10550/32116>.

Parker, J. y Herwood, D. (1998). The earth and beyond: developing primary teacher's understanding of astronomical events. International Journal of Science Education, 20, pp. 503-519. http://dx.doi.org/10.1080/0950069980200501

Pasachoff, J. M. (Septiembre de 2001). What Should Students Learn? The phisycs teacher, 39, pp. 381-383.

Pérez Sedeño, E. (2003). Las mujeres en la historia de la ciencia. Quark: Ciencia, medicina, comunicación y cultura (27). Disponible en línea: <http://quark.prbb.org/27/027060.htm> (consulta 3/30/2014).

Solbes, J. (2009). Dificultades de aprendizaje y cambio conceptual, procedimental y axiológico. Revista Eureka sobre Enseñanza y Divulgación de las Ciencias, 6(1), pp. 2-20.

Solbes, J. y Palomar, R. (2011). ¿Por qué resulta tan difícil la comprensión de la astronomía a los estudiantes? Didáctica de las ciencias experimentales y sociales 25, pp. 187-211.

Solbes, J. y Palomar, R. (2013). Dificultades del aprendizaje de la astronomía en secundaria. Revista Brasileira de Ensino de Física, 35(1), p. 1401. http://dx.doi.org/10.1590/S1806-11172013000100016

Solbes, J. y Traver, M. (2003). Against negative image of science: history of science in the physics $\&$ chemistry education. Science \& Education, 12, pp. 703-717. http://dx.doi.org/10.1023/A:1025660420721

Solbes, J. y Vilches, A. (1997). STS interactions and the teaching of physics and chemistry. Science Education, 81(4), pp. 377-386. http://dx.doi.org/10.1002/(SICI) 1098-237X(199707)81:4<377::AID-SCE1>3.0.CO;2-9

Solbes, J., Montserrat, R. y Furió, C. (2007). El desinterés del alumnado hacia el aprendizaje de la ciencia: implicaciones en su enseñanza. Didáctica de las Ciencias Experimentales y Sociales, 21, pp. 91-117.

Trumper, R. (2001). A cross-age study of junior high school students' conceptions on basic astronomy concepts. International Journal of science education, 23(11), pp. 1111-1123. http://dx.doi.org/10.1080/09500690010025085

Trundle, K. C., Atwood, R. K. y Chistopher, J. E. (2007). Fourth Grade Elementary Students' Conceptions on Standards-Based Lunar Concepts. International Journal of Science Education, 29(25), pp. 595-616. http://dx.doi.org/10.1080/09500690600779932

Vega, A. (2001). Tenerife tiene seguro de Sol (y de luna): Representaciones del profesorado de primaria acerca del día y la noche. Enseñanza de las Ciencias, 19(1), pp. 31-44.

ZuZA, K. y Alduncin, J. A. (2009). ¿Se puede conseguir que los estudiantes se aficionen a la astronomía si no pueden disfrutar del cielo nocturno? Alambique 61, pp. 65-72. 


\title{
Evaluation of an instruction for teaching and learning of astronomy in secondary school
}

\author{
Rafael Palomar \\ Colegio El Prat. LLíria. España. \\ rafa.palomar@gmail.com \\ Jordi Solbes \\ Departamento de didáctica de las ciencias. Universitat de València. España \\ Jordi.solbes@uv.es
}

This work is a continuation of a previous investigation in order to evaluate the astronomical knowledge of a group of first year high school students (17 years old). This group completed the subject Ciencias para el Mundo Contemporáneo (Sciences for the Contemporary World). The students obtained very low scores. A detailed analysis of the results allowed us to detect a list of learning difficulties. Furthermore, the analysis of the most popular texts on this subject revealed that books are not taking into account the students' difficulties.

Based on these results, a didactic proposal was designed to resolve this situation and reach the objectives, taking into account the difficulties in science teaching and especially in astronomy. The program of activities has been developed in a previous work. Besides, the proposal is published on one of the authors' website (www. astrodidactica.com, 'Science for the Contemporary World' section), a site that collects proposals and materials for teaching astronomy.

In our proposal, the contents are treated from the point of view of concepts, with a contextualized science, in its full complexity; procedures, since astronomy is an observational science; and attitudes, which show the complex relationship between science, technology and society.

In order to confirm how the proposal contributes to improve the difficulties of student learning, a questionnaire was used, although it will not be described in this article as it was the object of a previous investigation. The questionnaire was applied to 99 students before teaching the lesson (pre-test), and seven days after the completion of the lesson (post-test). Finally, a statistical analysis was performed with different tests according to the situation to be analyzed.

The proposal obtained statistically significant results for all items, with a large effect size of majority (7), medium for 4 . The item 8 , which relates to the interpretation of the scales in the solar system, obtained a small effect size. The latest will require special attention in future treatments, due to the difficulty of handling distances higher than the human scale. Considering the size of the effect generated by the contribution of all items, the result is a larger effect size $(\mathrm{d}=1.13)$.

By means of relevant statistical tests, it has been proven how students of both modalities of high school start the subject with similar astronomical knowledge, despite the fact that the students of the science and technology modality studied astronomy content in the previous year. After validating the statistically significant improvement in both modalities, it was observed that students of science and technology have progressed more than those of humanities and social sciences. For this last group, there has been no improvement in the items related to aspects like relationship between science, technology and society, as well as changes in the reference system of the observer and scales. It seems that science and technology students are more skilled on these items. 
